\title{
SENSITIVITY OPTIMIZATION AT THE DENSIFICATION GPS NETWORKS FOR MONITORING CRUSTAL MOVEMENTS
}

DOI: http://dx.doi.org/10.18509/GBP.2017.02

UDC: 528.8:004.77]:519.863

551.3:550.8.04

\author{
Haluk Konak \\ Pakize Küreç Nehbit \\ Cankut Dağdal İnce \\ Kocaeli University, Turkey
}

\begin{abstract}
Densification GPS Networks must provide the precision and reliability requirements. At the same time, these networks should be able to detect the possible earth crust movements. If the total weight of observation at a point raises, the sensitivity capacity of the point increases with the same rate. However, controllability of these observations weakens relative to neighboring observations. This paradox is a fundamental design problem that it must be resolved in the evaluation phase of geodetic networks. In this study, an improvement strategy has been recommended as a solution to the design problem.
\end{abstract}

Keywords: External Reliability, Sensitivity, Optimization, Crustal Movements, Densification GPS Networks.

\section{INTRODUCTION}

Densification GPS Networks evaluated in different epochs allow monitoring of crustal movement in the region, updating of velocity fields and determining displacements in the network's points. Therefore, the Permanent or the Fundamental Densification GPS/GNSS Networks must provide the precision and reliability requirements. At the same time, these networks should be able to detect crustal movements with sufficient accuracy. In other words, the External Reliability and the Sensitivity criteria should have a good distribution. External reliability can be described as effects on the coordinate unknowns of the undetectable gross errors by using hypothesis testing. The minimum value of undetectable gross errors in the adjusted coordinate differences between epochs is named sensitivity level for the geodetic network. If the total weights of observations at a GPS point raises, the sensitivity capacity of this point increases with the same rate. However, reliabilities of these observations get quite weak. The reliability and the sensitivity criteria are two basic criteria which complete each other. However, due to the obtained GPS observations with different accuracy in the different epochs, these criteria affect each other in through the opposite direction. For this reason, both the external reliability values and the sensitivity levels should be obtained in good level. This paradox is an important design matter for the geodetic networks. The solution of the matter is the main idea of the study. For this purpose, an optimization strategy is recommended in this study. This strategy is applied to a real densification test network called IZDOGAP GPS Network (Fig. 1). 


\section{OPTIMIZATION STRATEGY}

The location of any measurement in the observation plan and its precision directly affect the controllability of the observation. Therefore, it is necessary that an observation must control with neighboring observations on an equal level. Nevertheless, in the Densification GPS Networks, the total weight of observations at a station point is not always obtained on an equal level for each epoch [1]. If total weight of observations at a station point is higher, sensitivity level of the point also gets better. However, controllability and external reliability values of mentioned observations get weak. In other words, the good sensitivity level does not guarantee the appropriate reliability distribution in every time [2]. This situation is an important design matter. The optimization strategy is recommended to resolve the basic design problem. The main purpose of this study is to obtain an optimal sensitivity and external reliability distribution in networks for monitoring crustal movements.

a. The Creation of the Design Matrix: An appropriate observation plan is designed. The Delaunay triangulation, expert opinion, simulation methods or the second order designed process can be used to provide the stable geometric shape or the observation plan [3].

b. Analysis of the Geometric Shape: At designed observation plan, observation weights are accepted equal $(\mathbf{P}=\mathbf{E})$ for geometric shape analysis. In each epoch, observations are evaluated with the Gauss-Markoff Model using the least squares method:

$$
\mathbf{v}=\mathbf{A x}-\mathbf{l} ; \mathbf{P}=\mathbf{E}
$$

where $\mathbf{A}$ is the design matrix, $\mathbf{l}$ is the observation vector, $\mathbf{x}$ is unknown parameters and $\mathbf{v}$ is correction vector. The external reliability values $\left(\delta_{j}\right)$ are computed with:

$$
\delta_{j}^{2}=\delta_{0}^{2} \frac{\mathbf{e}_{j}^{T} \mathbf{P} \mathbf{Q}_{\tilde{l} \tilde{l}} \mathbf{P} \mathbf{e}_{j}}{\mathbf{e}_{j}^{T} \mathbf{P} \mathbf{Q}_{v v} \mathbf{P e} \mathbf{e}_{j}}
$$

where $\delta_{0}^{2}=F\left(\alpha_{0}, \beta_{0}, h, \infty\right)$ is a limit value of non-centrality parameter, $\mathbf{e}_{j}$ is a design vector for $\mathbf{j}^{\text {th }}$ observation, $\mathbf{Q}_{\tilde{l} \tilde{l}}$ is the cofactor matrix of adjusted observations and $\mathbf{Q}_{v v}$ is the cofactor matrix of residuals [3]. Thus, the strong and weak properties of the geometric shape are reviewed.

In a sufficient level designed Geodetic Network, external reliability levels $\left(\delta_{0 i}\right)$ remain between 6-10 units. To obtain a sufficient network, either the other network designs or the optimization methods could be experimented by return to the first step. For this aim, an analytical approximation, namely second order design problems, should be proposed for the optimal observation plan and optimal dispersion of their reliabilities. However, the primary subject of the study is not the development of any second order design $[4,5$, 6].

c. A Priori Sensitivity Analysis: According to the observation plan designed, the geodetic observations are obtained and the network observations are evaluated for each epoch. At the end of the evaluation process, a priori sensitivity values of first two epochs of the geodetic network for $\Delta t=\sqrt{2}$-unit year are obtained. Adjusted coordinate values $\left(\hat{\mathbf{x}}^{1}, \hat{\mathbf{x}}^{0}\right)$ obtained from evaluation results of a geodetic network in any time interval depend on randomly distributed errors $\left.\mid \mathbf{d} \mathbf{l}=\left(\mathbf{l}^{1}, \mathbf{l}^{0}\right)\right]$ in the observation vector $\left(\mathbf{l}^{1}, \mathbf{l}^{0}\right)$. The difference vector between the adjusted coordinates obtained from $t_{0}$ and $t_{1}$ epochs can be described as:

$$
\mathbf{d}=\mathbf{x}^{1}-\mathbf{x}^{0}=\mathbf{N}^{+} \mathbf{A}^{\mathrm{T}} \mathbf{P d} \mathbf{l}
$$


the displacement vector (d) is arranged for 3D GPS Networks as:

$$
\left[\begin{array}{c}
\mathbf{d}_{1} \\
\hdashline \mathbf{d}_{2} \\
\cdot \\
\mathbf{d}_{n}
\end{array}\right]=\left[\begin{array}{cccc}
\mathbf{N}_{11} & \mathbf{N}_{12} & \cdot & \mathbf{N}_{1 n} \\
\hdashline \mathbf{N}_{21}^{-} & \mathbf{N}_{22}^{-} & \cdot & \mathbf{N}_{2 n}^{-} \\
\cdot & \cdot & \cdot & \cdot \\
\mathbf{N}_{n 1} & \mathbf{N}_{n 2} & \cdot & \mathbf{N}_{n n}
\end{array}\right]^{+}\left[\begin{array}{c}
\left(\mathbf{A}^{T} \mathbf{P d l}\right)_{1} \\
\left(\mathbf{A}^{T} \mathbf{P d l}\right)_{2} \\
\cdot \\
\left(\mathbf{A}^{T} \mathbf{P d l}\right)_{n}
\end{array}\right]
$$

this equation can be written for a single GPS station (i) as:

$$
\mathbf{d}_{i}=\ddot{\mathbf{N}}_{i} \mathbf{A}^{T} \text { Pdl }
$$

and then according to the law of variance-covariance propagation, the cofactor matrix of the displacement vector at any station point is calculated separately with the following equations:

$$
\mathbf{Q}_{d_{i} d_{i}}=\ddot{\mathbf{N}}_{i} \mathbf{A}^{T} \mathbf{P} \mathbf{Q}_{l l} \mathbf{P} \mathbf{A} \ddot{\mathbf{N}}_{i}^{T}=\ddot{\mathbf{N}}_{i} \mathbf{N} \ddot{\mathbf{N}}_{i}^{T}
$$

Local sensitivity level ( $\left.\|d\|_{\min }\right)$ can be obtained separately by examining eigenvalues of the weight matrix $\left(\mathbf{Q}_{d_{i} d_{i}}^{-1}\right)$ of each station point.

$$
\|d\|_{\min }=\frac{\delta_{0} \sigma}{\sqrt{\lambda_{\max }}}
$$

The epoch providing sensitivity values with possible good and homogeneous is defined as the objective function $\left(\boldsymbol{\Omega}=\min \left\{\operatorname{trace}\left(\mathbf{Q}_{x x}^{0}\right) ; \operatorname{trace}\left(\mathbf{Q}_{x x}^{1}\right)\right\}\right)$ for the other epoch [7,3].

d. The Improvement of External Reliability Distribution: The distributions of external reliability obtained for two epochs are reviewed. A Robust Weighting method is used to reduce the impact of poor controllability observations. The weights of the observations exceeding the threshold value (c) are reduced by using a proper robust weighting process and then a controllable external reliability distribution is obtained.

In each GPS baseline, robust weight function $\left(\mathbf{w}_{j}\right)$ is computed for the external reliability value $\left(\delta_{j_{\max }}\right)$ passing the threshold value with the following equation.

$$
\mathbf{w}_{j}=e^{\frac{\delta_{j_{\max }-c}}{0.5 * c}}
$$

The cofactor matrix of observations is reweighted as:

$$
\overline{\mathbf{Q}}_{l l_{j}}=\mathbf{Q}_{l l_{j}} * \mathbf{w}_{j}
$$

The observation plan, which provides the best sensitivity option from both epochs, is confirmed as the objective function for sensitivity improvements and a posteriori sensitivity analysis.

e. The Improvement of the Sensitivity Distribution: After the reliability improvement step, the epoch with poor sensitivity distributions is improved according to epoch selected as the objective function $\left(\mathbf{Q}=\min \left\{\operatorname{trace}\left(\mathbf{Q}_{x x}^{0}\right) ; \operatorname{trace}\left(\mathbf{Q}_{x x}^{1}\right)\right\}\right)$. The scaling coefficient $\left(\Lambda_{i}\right)$, which minimizes the global approximation criteria $\left(\Delta \mathbf{d}^{T} \Delta \mathbf{d} \Rightarrow \min\right.$. $)$ related to second order design, is computed by using cofactor matrices for each station point.

$$
\Lambda_{i}=\frac{i z\left[\mathbf{Q}_{x i x i}^{k} \mathbf{Q}_{x i x i}^{k}\right]}{i z\left[\mathbf{Q}_{x i x i}^{k} \mathbf{Q}\right]}, k=\{0 \vee 1\}
$$

During the improvement process, a threshold value $\left(\Lambda_{s}=3,5,7,10 \ldots\right)$ representing the network is determined for scale coefficients. The improvement has been applied for the 
observations only exceeding the threshold value. The observation weights are rescaled using with the scaling coefficient. The network option, which provides objective function determined, should fulfill precision requests in homogeneous and isotropic structure. Thus, in the end of the evaluation processes of the first two epochs, these requests are maintained.

f. A Posteriori Sensitivity Analysis: Improved network results for first two epochs are combined and evaluated as a multivariate network. In this evaluation process, an optimal distribution is reached for the posteriori sensitivity values (Fig. 2, Table 2).

The velocity vector for the multivariate networks is written as:

$$
\dot{\mathbf{x}}_{i}=\frac{1}{\Delta t}\left[\ddot{\mathbf{N}}_{i}^{1}\left(\mathbf{A}^{T} \mathbf{P l}\right)^{1}-\ddot{\mathbf{N}}_{i}^{0}\left(\mathbf{A}^{T} \mathbf{P} \mathbf{l}\right)^{0}\right]
$$

the cofactor matrix of the velocity vector can be obtained with the following equations:

$$
\mathbf{Q}_{\dot{x}_{i} \dot{x}_{i}}=\frac{\left(\ddot{\mathbf{N}}_{i} \mathbf{N} \ddot{\mathbf{N}}_{i}^{T}\right)^{1}+\left(\ddot{\mathbf{N}}_{i} \mathbf{N} \ddot{\mathbf{N}}_{i}^{T}\right)^{0}}{\Delta t^{2}}
$$

and the weight matrix of each station point is computed as:

$$
\mathbf{P}_{\dot{x}_{i}}=\mathbf{Q}_{\dot{x}_{i} \dot{x}_{i}}^{-1}
$$

The success rates of the results obtained are reviewed and compared with results obtained from the original experimental data. Finally, in this improvement strategy, the sensitivity values of network option approved as the objective function must be used as the basic criterion.

\section{NUMERICAL APLICATION}

In this study, an observation plan designed for a real densification GPS Network is used. At the same time this network has supported to a scientific research project. The network having 106 GPS stations located at Kocaeli/Turkey was established to monitor crustal movement effects and to control gas pipelines. The network called as Kocaeli IZDOGAP GPS Network surrounds the Izmit-Sapanca fault which is west part of the North Anatolian Fault Zone. The north part of the fault is located in the Eurasian Plate and the south part of the fault is located in the Anatolian Plate. In the network area, Kocaeli/Gölcuik Earthquake having 7.4 magnitudes has occurred in 1999. The south part of the GPS network has movement of approximately $1-2 \mathrm{~cm}$ per year from east to west direction.

The network has been measured and evaluated, in 2009 and 2010 respectively. Before the improvement process, IZDOGAP Densification GPS Network is reviewed in terms of geometric shape and level of a priori sensitivity. When the network observations is evaluated under the $\mathrm{P}=\mathrm{E}$ condition, it is seen that both epochs are controlled on equivalent levels according to priori sensitivity and geometric shape. It is proved that the observation plan and the geometrical shape determined with optimization process are quite strong. When the a priori sensitivity and the external reliability distributions are analyzed, it is observed that the sensitivity difference between two epochs in 73 point and the external reliability differences between two epochs in 43-47, 41-105 and 60-40 base lines are much. Similar behaviors can be seen in other parts of the network too (Fig. 2a and 2b). A selected area which is restricted with only around 47 and 73 points will be sufficient to present the contributions of recommended strategy (Fig. 1). 
Table 1. The external reliability and sensitivity optimization results

\begin{tabular}{|c|c|c|c|c|c|c|c|c|c|}
\hline \multirow{3}{*}{ P. Num. } & \multicolumn{3}{|c|}{ Epoch 2009.370} & \multicolumn{6}{|c|}{ Epoch 2010.496} \\
\hline & \multicolumn{2}{|c|}{ Robust Model } & \multirow{2}{*}{$\begin{array}{c}\text { For } \\
\text { Common } \\
252 \text { Base } \\
\text { Line } \\
\mathrm{d}_{\min }(\mathrm{cm}) \\
\end{array}$} & \multicolumn{2}{|c|}{ Robust Model } & \multirow{3}{*}{$\begin{array}{c}\text { For } \\
\text { Common } \\
252 \text { Base } \\
\text { Line } \\
\mathrm{d}_{\min }(\mathrm{cm}) \\
0.60\end{array}$} & \multicolumn{3}{|c|}{$\begin{array}{c}\Lambda \text { Norm ( } \\
\left.\Lambda_{s}=10\right)\end{array}$} \\
\hline & $\delta_{\max }$ & $\begin{array}{l}\mathrm{d}_{\min } \\
(\mathrm{cm})\end{array}$ & & $\delta_{\max }$ & $\begin{array}{l}\mathrm{d}_{\min } \\
(\mathrm{cm})\end{array}$ & & \multirow{2}{*}{$\begin{array}{c}\begin{array}{c}\mathrm{d}_{\min } \\
(\mathrm{cm})\end{array} \\
\mathbf{0 . 5 7} \\
\end{array}$} & \multicolumn{2}{|l|}{$\delta_{\max }$} \\
\hline $41(204)$ & 5.51 & 0.33 & 0.34 & 7.99 & $* 0.55$ & & & 8.95 & $\mathbf{x}$ \\
\hline 43 & 5.06 & 0.32 & 0.33 & 7.89 & $* 0.58$ & 0.58 & 0.58 & 9.57 & $\mathbf{x}$ \\
\hline 46 & 4.40 & 0.35 & 0.37 & 3.74 & 0.56 & 0.61 & 0.57 & 3.76 & + \\
\hline 47 & 4.46 & 0.38 & 0.39 & 7.89 & $* 0.70$ & 0.68 & 0.67 & 9.57 & $\mathbf{x}$ \\
\hline 52 & 5.83 & 0.39 & 0.40 & 5.44 & 0.54 & 0.55 & 0.54 & 5.72 & + \\
\hline 65 & 5.51 & 0.37 & 0.37 & 5.49 & 0.56 & 0.60 & 0.56 & 5.57 & + \\
\hline 70 & 2.85 & 0.38 & 0.44 & 5.50 & 0.55 & 0.67 & 0.63 & 6.36 & + \\
\hline $71(203)$ & 5.53 & 0.42 & 0.43 & 5.50 & 0.61 & 0.79 & 0.66 & 6.36 & + \\
\hline 75 & 4.06 & 0.34 & 0.35 & 5.10 & 0.57 & 0.61 & 0.57 & 5.06 & + \\
\hline 76 & 5.06 & 0.32 & 0.33 & 5.10 & 0.64 & 0.63 & 0.64 & 5.06 & + \\
\hline 77 & 4.49 & 0.35 & 0.35 & 4.13 & 0.66 & 0.71 & 0.66 & 4.16 & + \\
\hline 105 & 5.36 & 0.32 & 0.33 & 7.99 & $* 0.53$ & 0.60 & 0.56 & 8.95 & $\mathbf{x}$ \\
\hline 59 & 5.35 & 0.41 & 0.41 & 7.68 & $* 0.60$ & 0.71 & 0.58 & 6.45 & + \\
\hline 42 & 5.35 & 0.36 & 0.37 & 7.68 & $* 0.68$ & 0.68 & 0.59 & 6.45 & $\mathbf{x}$ \\
\hline 106 & 4.52 & 0.43 & 0.44 & 5.79 & 0.57 & 0.61 & 0.58 & 6.13 & + \\
\hline $23(201)$ & 3.45 & 0.33 & 0.34 & 4.56 & 0.68 & 0.69 & 0.68 & 4.53 & + \\
\hline 73 & 4.69 & 0.34 & 0.36 & 4.59 & 1.01 & 2.25 & 0.57 & 10.83 & $\mathbf{x}$ \\
\hline 74 & 4.69 & 0.34 & 0.35 & 4.59 & 0.64 & 0.88 & 0.57 & 10.83 & $\mathbf{x}$ \\
\hline 60 & 5.38 & 0.40 & 0.42 & 7.94 & $* 0.68$ & 1.23 & 0.63 & 8.81 & $\mathbf{x}$ \\
\hline 40 & 3.59 & 0.37 & 0.40 & 7.94 & $* 0.68$ & 1.70 & 0.61 & 8.81 & $\mathbf{X}$ \\
\hline 68 & 4.15 & 0.40 & 0.41 & 4.28 & 0.65 & 0.85 & 0.63 & 4.26 & + \\
\hline 69 & 5.04 & 0.42 & 0.42 & 3.65 & 0.58 & 0.72 & 0.60 & 4.92 & + \\
\hline 63 & 5.38 & 0.36 & 0.36 & 3.86 & 0.53 & 0.61 & 0.53 & 3.91 & + \\
\hline $\mathrm{SA}\left(\delta_{\max }\right)$ & 5.83 & & & 7.99 & & & & 10.83 & \\
\hline $\operatorname{IZDOGAP}\left(\delta_{\max }\right)$ & 8.00 & & & 8.00 & & & & 11.42 & \\
\hline $\begin{array}{r}\text { *: In Epoch } 2 \\
\delta_{\mathrm{ma}} \\
\text { IZDOGAP }\end{array}$ & $\begin{array}{l}\text {, the } \mathrm{C} \\
\text { rnal re } \\
=\text { max } \\
\mathrm{SA}: \mathrm{Se} \\
\text { ocaeli }\end{array}$ & $\begin{array}{l}\text { S statio } \\
\text { bility v } \\
4 X, \delta_{\Delta Y} \\
\text { cted are } \\
\text { DOGA }\end{array}$ & $\begin{array}{l}\text { having the } \\
\text { les. } \\
\left.S_{\Delta Z}\right\}_{i} \\
\text { GPS Netw }\end{array}$ & & $\begin{array}{l}(0 \leq \delta \\
(8 \leq \\
\left(\delta_{\max }\right.\end{array}$ & $\begin{array}{l}\operatorname{ax} \leq 8) \\
\max \leq 1 \\
\text { 15) }\end{array}$ & $\begin{array}{l}\text { ccessf } \\
\text {, Suff } \\
\text { ?) }\end{array}$ & $\begin{array}{l}(+) \\
\text { ient }(X)\end{array}$ & \\
\hline
\end{tabular}

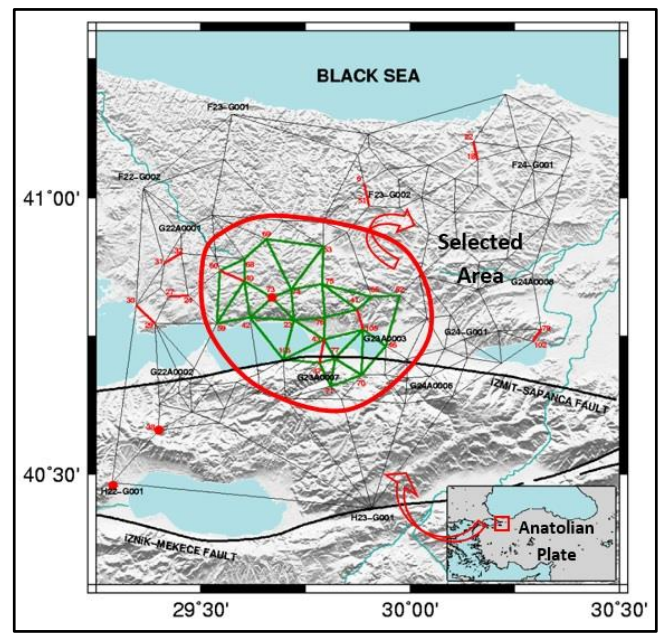

Figure 1. IZDOGAP GPS network and the selected area (red line: discussable external reliability; red point: discussable sensitivity). 


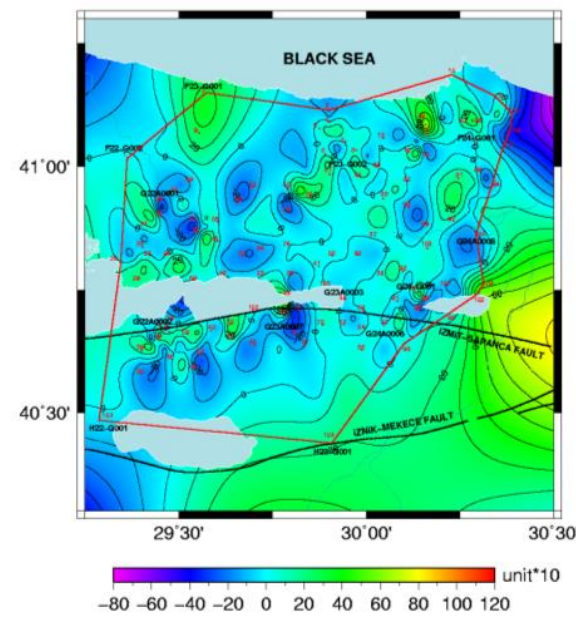

2a The difference map for the experimantel external reliability

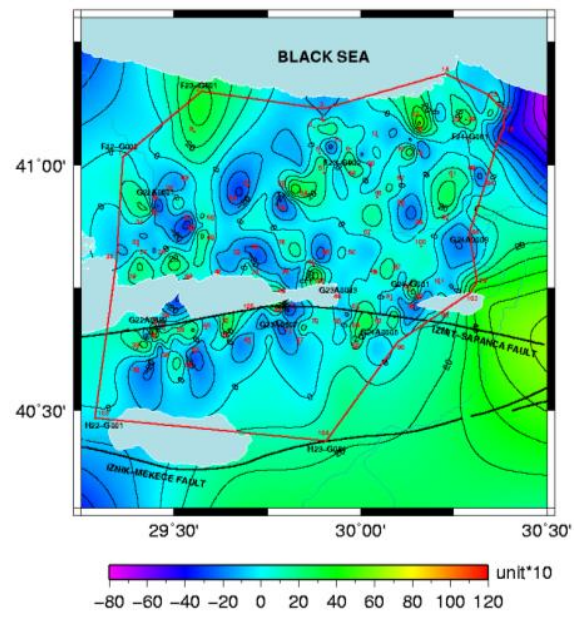

2c The difference map for the external reliability with robust model

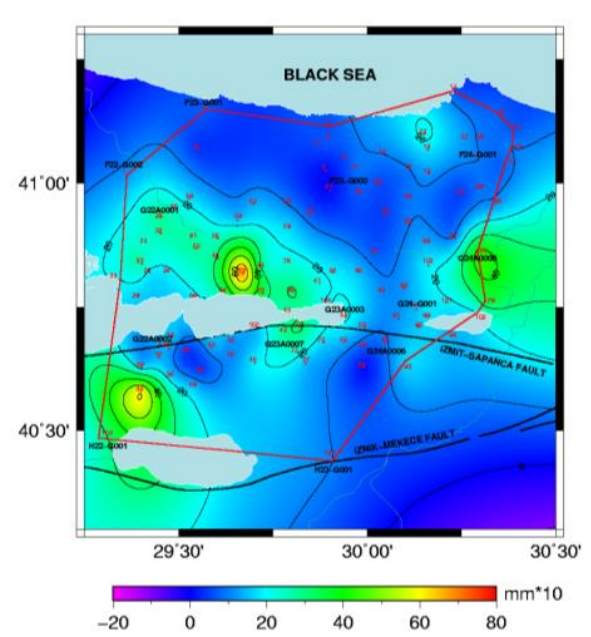

$\mathbf{2 b}$ The difference map for a priori sensitivity

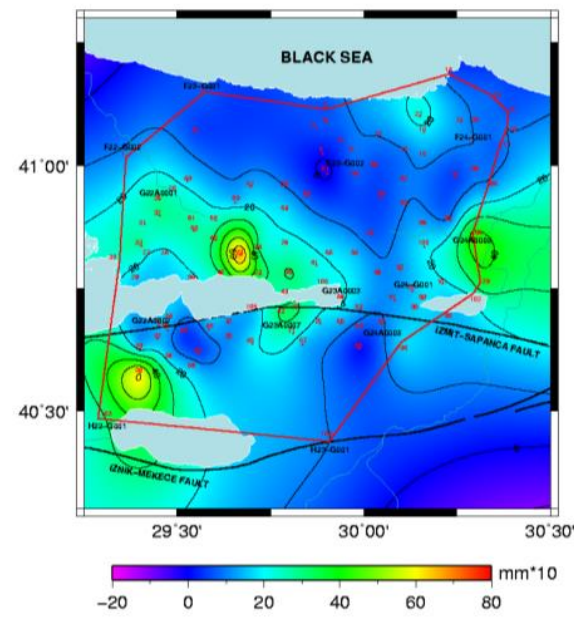

2d The difference map for a priori sensitivity with robust model

Figure 2. The difference maps of the sensitivity and the external reliability

The weights of the observations exceeding the threshold value $(\mathrm{c}=8)$ of the external reliability $\left(\delta_{\max }>\right.$ c) in epochs 2009 and 2010 are reduced by using the robust weighting method. Thus, the external reliability distribution of IZDOGAP GPS Network is improved. At the end of the improvement process; weights of 9 GPS observations (6040, 47-43, 6-51, 22-18, 31-32, 79-102, 27-24, 29-30, 41-105) in 2010 epoch are improved. On the other hand, only one GPS observation (103-38) located out of the selected area is improved for epoch 2009. It is observed significant improvements for the external reliability values of epoch 2010 , but some distortions for the sensitivity distribution are determined (Fig. 2c and 2d).

The observation weights in epoch 2009 are a design representing the homogeneous and isotropic network requests. For this reason, both the external reliability and the sensitivity distributions are quite homogeneous and consistent. The sensitivity values of epoch 2010 are approximated to the sensitivity values of 2009 by using the sensitivity improvement process. If the threshold value of the scale coefficients $\left(\Lambda_{s}\right)$ is selected 10 , both the sensitivity and the reliability distributions in epoch 2010 are improved in a controllable 
and stable structure. Therefore, it is recommended that the threshold value is 10 for the sensitivity improvement (Table 1).

\section{CONCLUSIONS and RECOMMENDATIONS}

It is expected that each epoch of GPS/GNSS networks has the homogeneous and isotropic distributions in terms of both the external reliability and the sensitivity. For this reason, the external reliabilities and the sensitivities should be reviewed once more in each epoch of these networks. The base lines having divergence from the external reliability distributions should be improved in each epoch. Furthermore, if there are any network points which disrupt the sensitivity distribution, total precision of the observations in these points should be made similar to other network points. According to the results obtained from improved İZDGAP GPS Network;

- Locations of problematic observations and stations can be estimated in each epoch and then the sensitivity distribution can be developed by using the improvement strategy.

- Before the land work is finished in an epoch, an appropriate observing time and additional observations can be planned by using the strategy.

- In most importantly, a posteriori sensitivity values with homogeneous distribution for each epoch can be guaranteed.

Table 2. The sensitivity distributions in selected area.

\begin{tabular}{|c|c|c|c|c|c|c|}
\hline \multirow[b]{3}{*}{ P. Num } & \multicolumn{3}{|c|}{ Experimental Weights } & \multicolumn{3}{|c|}{ Optimal Weights } \\
\hline & \multicolumn{2}{|c|}{ A Priori } & \multirow{2}{*}{$\begin{array}{c}\text { A } \\
\text { Posteriori } \\
\Delta t \\
\mathrm{~d}_{\min }(\mathrm{cm})\end{array}$} & \multicolumn{2}{|c|}{ A Priori } & \multirow{2}{*}{$\begin{array}{c}\mathrm{A} \\
\text { Posteriori } \\
\Delta t \\
\mathrm{~d}_{\min }(\mathrm{cm})\end{array}$} \\
\hline & $\begin{array}{c}2009.370 \\
\mathrm{~d}_{\min } \\
(\mathrm{cm})\end{array}$ & $\begin{array}{c}2010.496 \\
\mathrm{~d}_{\min } \\
(\mathrm{cm})\end{array}$ & & $\begin{array}{c}2009.370 \\
\mathrm{~d}_{\min } \\
(\mathrm{cm})\end{array}$ & $\begin{array}{c}2010.496 \\
\mathrm{~d}_{\min } \\
(\mathrm{cm})\end{array}$ & \\
\hline * $41(204)$ & $\mathbf{0 . 3 3}$ & 0.54 & $\mathbf{0 . 5 8}$ & $\mathbf{0 . 3 3}$ & 0.54 & 0.58 \\
\hline$* 43$ & 0.32 & 0.58 & 0.59 & 0.32 & 0.57 & 0.58 \\
\hline 46 & 0.35 & 0.56 & 0.59 & 0.35 & 0.56 & 0.59 \\
\hline$* 47$ & 0.38 & 0.67 & 0.69 & 0.38 & 0.70 & 0.71 \\
\hline 52 & 0.39 & 0.54 & 0.59 & 0.39 & 0.54 & 0.59 \\
\hline 65 & 0.37 & 0.56 & 0.60 & 0.37 & 0.56 & 0.60 \\
\hline 70 & 0.38 & 0.55 & 0.60 & 0.38 & 0.55 & 0.60 \\
\hline $71(203)$ & 0.42 & 0.61 & 0.67 & 0.42 & 0.61 & 0.67 \\
\hline 75 & 0.34 & 0.56 & 0.59 & 0.34 & 0.56 & 0.59 \\
\hline 76 & 0.32 & 0.64 & 0.63 & 0.32 & 0.63 & 0.63 \\
\hline 77 & 0.35 & 0.65 & 0.66 & 0.35 & 0.65 & 0.66 \\
\hline$* 105$ & 0.32 & 0.52 & 0.55 & 0.32 & 0.52 & 0.55 \\
\hline * 59 & 0.41 & 0.60 & 0.64 & 0.41 & 0.57 & 0.62 \\
\hline$* 42$ & 0.36 & 0.68 & 0.69 & 0.36 & 0.58 & 0.61 \\
\hline 106 & 0.43 & 0.57 & 0.64 & 0.43 & 0.56 & 0.63 \\
\hline $23(201)$ & 0.33 & 0.68 & 0.68 & 0.33 & 0.67 & 0.66 \\
\hline$* * 73$ & 0.34 & 1.01 & 0.96 & 0.34 & 0.54 & 0.58 \\
\hline 74 & 0.34 & 0.64 & 0.65 & 0.34 & 0.53 & 0.57 \\
\hline$* 60$ & 0.40 & 0.67 & 0.70 & 0.40 & 0.63 & 0.67 \\
\hline$* 40$ & 0.37 & 0.68 & 0.69 & 0.37 & 0.60 & 0.63 \\
\hline 68 & 0.40 & 0.65 & 0.68 & 0.40 & 0.62 & 0.66 \\
\hline 69 & 0.42 & 0.58 & 0.64 & 0.42 & 0.56 & 0.63 \\
\hline 63 & 0.36 & 0.53 & 0.57 & 0.36 & 0.50 & 0.55 \\
\hline
\end{tabular}




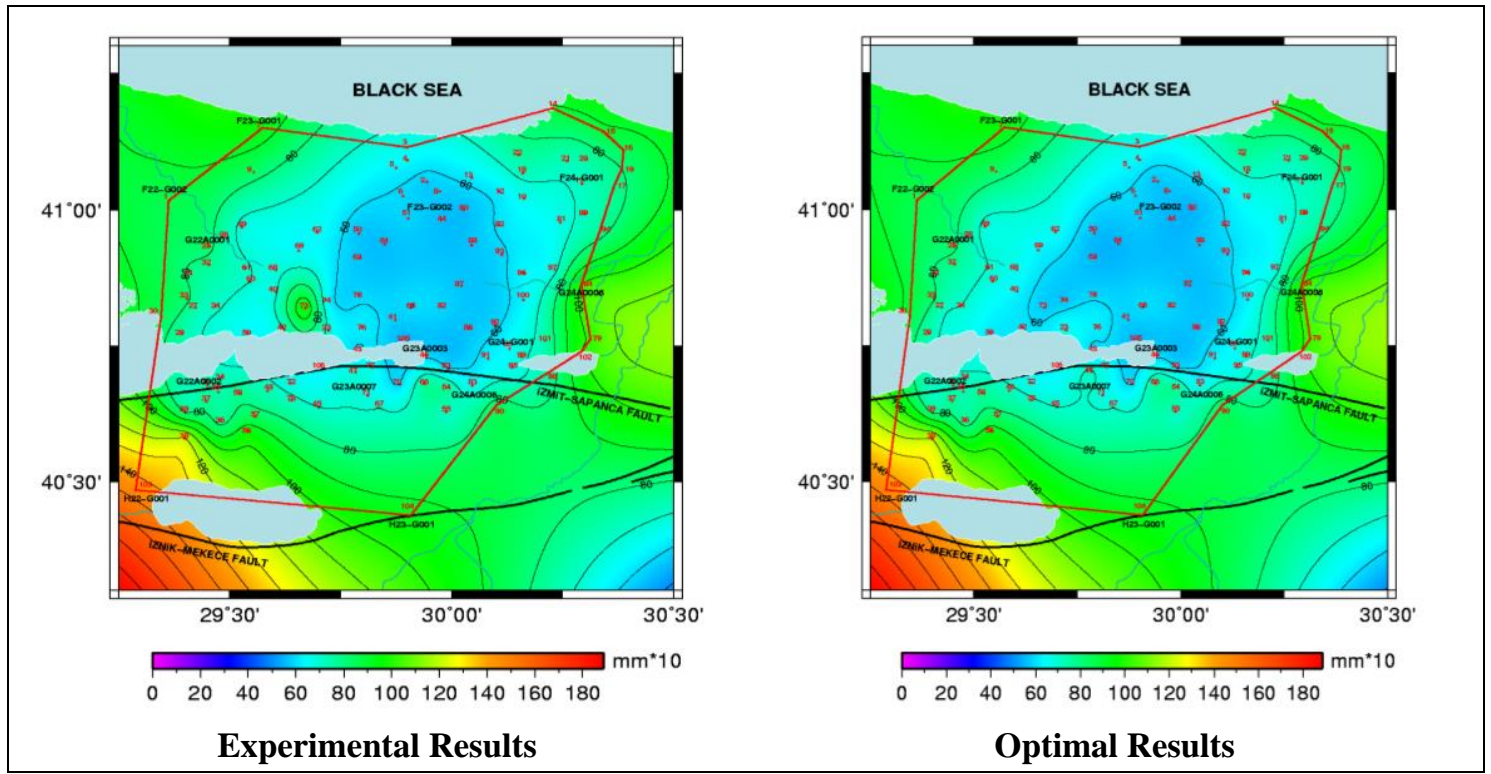

Figure 3. A posteriori sensitivity maps for $\Delta t$

\section{REFERENCES}

[1] Konak, H., Dilaver, A., and Öztürk, E. Effects of Observation Plan and Precision on the Duration of Outliers Detection and Fuzzy Logic: A Real Network Application, Survey Review, UK, October, 298, 331-341, 2005.

[2] Hsu, R., Lee, H. C., and Kao, S. P. Statistical Tests for Discerning Differences of Robustness of Horizontal Geodetic Networks due to Different Approaches. Journal of the Chinese Institute of Engineers. Taiwan, 31, 71-80, 2008.

[3] Küreç, P. and Konak, H. A priori sensitivity analysis for densification GPS networks and their capacities of crustal deformation monitoring a real GPS network application, Nat. Hazards Earth Syst. Sci., 14, 1299-1308, 2014.

[4] İZDOGAP. Monitoring of IZGAZ-Natural Gas Infrastructure using National Geodetic Networks and Land Information Systems (IZDOGAP). Technical Reports (Turkish). Ed. Haluk Konak. A Scientific Research Project, Kocaeli University,Turkey, 2011.

[5] Konak, H., Optimization of the Surface Network. Doctoral Thesis (Turkish), KTU, Trabzon, 1995.

[6] Schmitt, G. Second Order Desing: Optimization and Desing of Geodetic Networks. Edited by E. W. Grafarend and F. Sanso. Springer Verlag. Berlin Heidelberg New York Tokyo. e-ISBN-13: 978-3-642-70659-2. 74-121, 1985.

[7] Hsu, R. and Hsiao, K. Pre-Computing The Sensitivity of a GPS Station for Crustal Deformation Monitoring, Journal of the Chinese Institute of Engineers, 12-15, 2002. 